\title{
Comparison of different methods for evaluation of wind turbine power production based on wind measurements
}

\author{
Valerijs Bezrukovs ${ }^{1,2,}{ }^{*}$, Aleksejs Zacepins ${ }^{1}$, Vladislavs Bezrukovs ${ }^{2}$, and Vitalijs Komasilovs ${ }^{1}$ \\ ${ }^{1}$ Institute of Physical Energetics, 21 Aizkraukles Str., Riga LV-1006, Latvia \\ ${ }^{2}$ Ventspils University College, 101 Inzenieru Str., Ventspils LV-3601, Latvia
}

\begin{abstract}
Investigations of the wind shear up to the height of $200(\mathrm{~m})$ on the Latvian coast of the Baltic Sea have been carried out using a Pentalum SpiDAR laser measuring complex. Based on wind speeds measurements for three levels - 30, 40 and $50(\mathrm{~m})$, assessment of the operational efficiency of the wind turbines for heights 100, 140 and $180(\mathrm{~m})$ have been performed. For comparison, this analysis involves five different approaches: the Rayleigh frequency distribution, three different Weibull frequency distributions and method based on approximation of the cubic wind speed. Results are compared with measurements on the corresponding heights.
\end{abstract}

\section{Introduction}

The methods of analysis are based on extrapolation of the results obtained by measurements of the wind speed and standard deviation $(S D)$, and at modelling the probability density curve of wind speed function $F(V)$ using Rayleigh's models and Weibull's parameters $k$ (shape factor) and $c$ (scale factor) [1].

As an example, it is taken the operating characteristics of a $2300(\mathrm{~kW})$ Enercon E-82 wind turbines (WTs) [2] and have shown to what extent the precision of the calculation results obtained for the WT operational efficiency at different heights depends on the choice of the model. The proper choice of the model would enable calculation of the annual electricity production (AEP) by generators, making it possible to estimate the efficiency of WT offered for the use of a particular WT type.

\section{The results of wind shear investigation}

Figure 1 shows the map of disposition of a laser measuring complex Pentalum SpiDAR in the territory of Latvia on the Baltic Sea coast [3]. The measuring complex is installed in the territory of the Ventspils International Radio-Astronomy Centre at the height of $13.2(\mathrm{~m})$ above ground level and at a distance of $2.5(\mathrm{~km})$ from the seashore on a platform with coordinates N $57^{\circ} 33^{\prime} 12.00^{\prime \prime}$ and E $21^{\circ} 51^{\prime} 16.00^{\prime \prime}$.

The Pentalum SpiDAR performs 5 (s) measurements of wind speed and direction for ten height levels: $30,40,50,80$, $100,120,140,160,180$ and $200(\mathrm{~m})$. Apart from that, the

\footnotetext{
* e-mail: elmag@inbox.lv
}

records are made for pressure, humidity, and temperature of the air. Web system is developed for retrieving data from SpiDAR ftp server.

Figure 2 displays the curves of average monthly wind speed values, $V_{\text {avg }}$, which are calculated by averaging the bins, i.e. the measured wind speed values $V$ accumulated within $10 \mathrm{~min}$ in the time period $T$ from 01.03 .2014 to 01.03.2015 using the laser measuring complex Pentalum SpiDAR at the heights 30, 50, 100, 140 and 180 (m) above ground.

Figure 3 demonstrates the distribution curves of the average wind speed $V_{a v g}$ and the average cubic wind speed $V_{\text {avg cub }}(\mathrm{m} / \mathrm{s})$ values along with the $S D$ value for the wind shear up to the height of $200(\mathrm{~m})$. These values have been calculated based on analysis of the data accumulated during 12 months. The distribution of the obtained wind shear values is well approximated by the expression:

$$
V_{\text {avg }}=V_{\text {avg } h_{r}}\left(\frac{h}{h_{r}}\right)^{\alpha}(\mathrm{m} / \mathrm{s}),
$$

where

$h_{r}$ is the height of wind speed measurement $(\mathrm{m})$,

$h$ is the height of WT location (m),

$\alpha$ is the approximation coefficient.

Using the force function for approximation of the measurement results, expression (1) will assume the form [1]:

$$
V_{\text {avg }}=0.84 h^{0.45}(\mathrm{~m} / \mathrm{s}) .
$$

The distribution curve of $S D$ values is approximated using the exponential function which for the measurement results obtained will be:

$$
S D=1.649 e^{0.003 h} .
$$




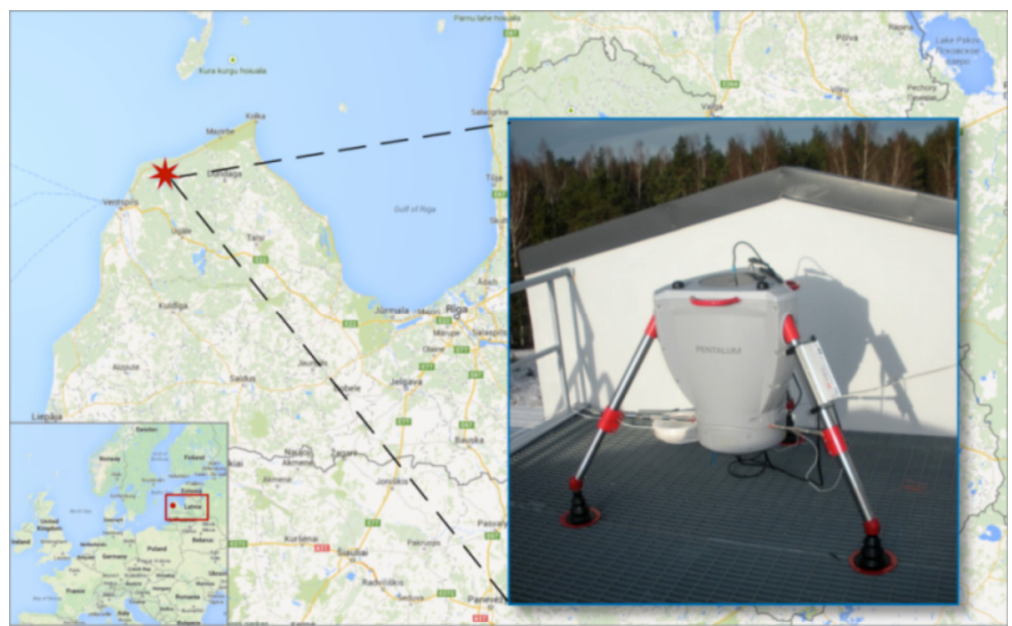

Fig. 1. Laser measuring complex Pentalum SpiDAR installed on the Baltic Sea coast at a height of $13.2(\mathrm{~m})$ above ground level and at a distance of $2.5(\mathrm{~km})$ from the sea coast.

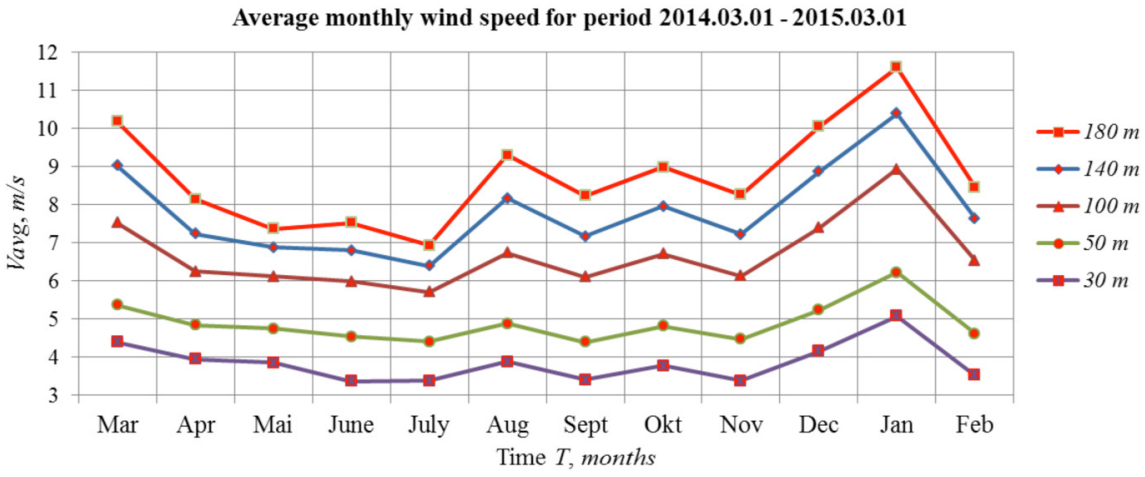

Fig. 2. Distribution curves of the average wind speed values $V_{\text {avg }}(\mathrm{m} / \mathrm{s})$ obtained using the laser measuring complex Pentalum SpiDAR for the heights $30,50,100,140$ and $180(\mathrm{~m})$ above ground in the measurement time $T$.

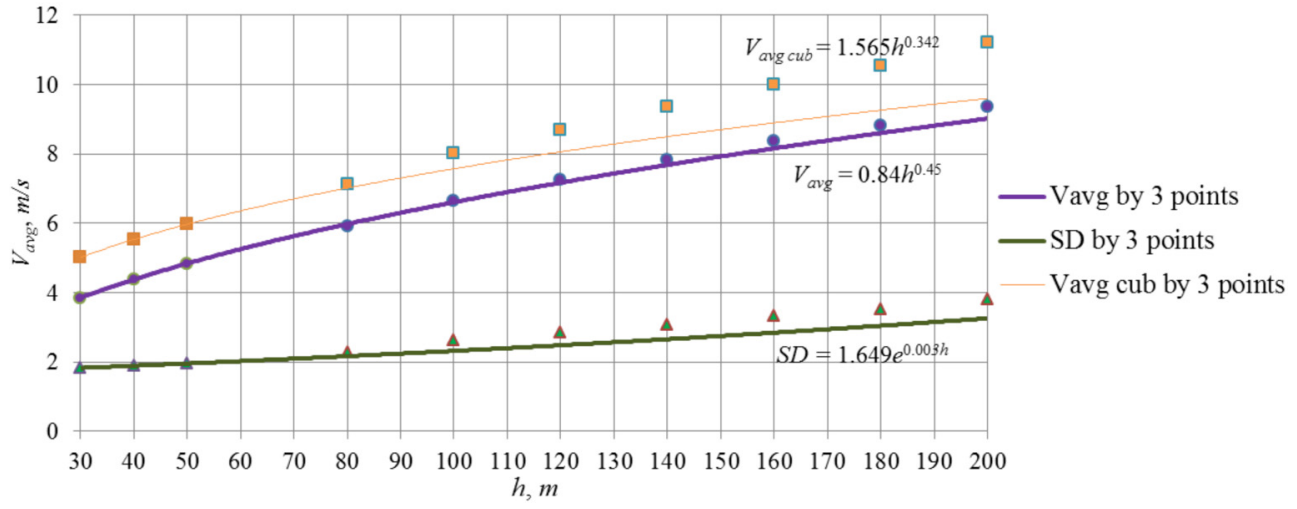

Fig. 3. Approximation curves for the measured values of wind speed $V_{\text {avg }}, V_{\text {avg cub }}(\mathrm{m} / \mathrm{s})$, and for the standard deviation, $S D$, depending on height $h$ by three points for heights of 30, 40 and $50(\mathrm{~m})$ above ground.

To approximate the values of cubic wind speed the force function described by the equation [2] can be used:

$$
V_{\text {avg cub }}=1.565 h^{0.342}(\mathrm{~m} / \mathrm{s}) .
$$

For adequate comparison of the modelling methods and their further analysis, at determination of expressions for approximating functions (2), (3) and (4), the values corresponding to the three heights $(30,40$ and $50(\mathrm{~m}))$ are used. In these cases, the regularities describing the 


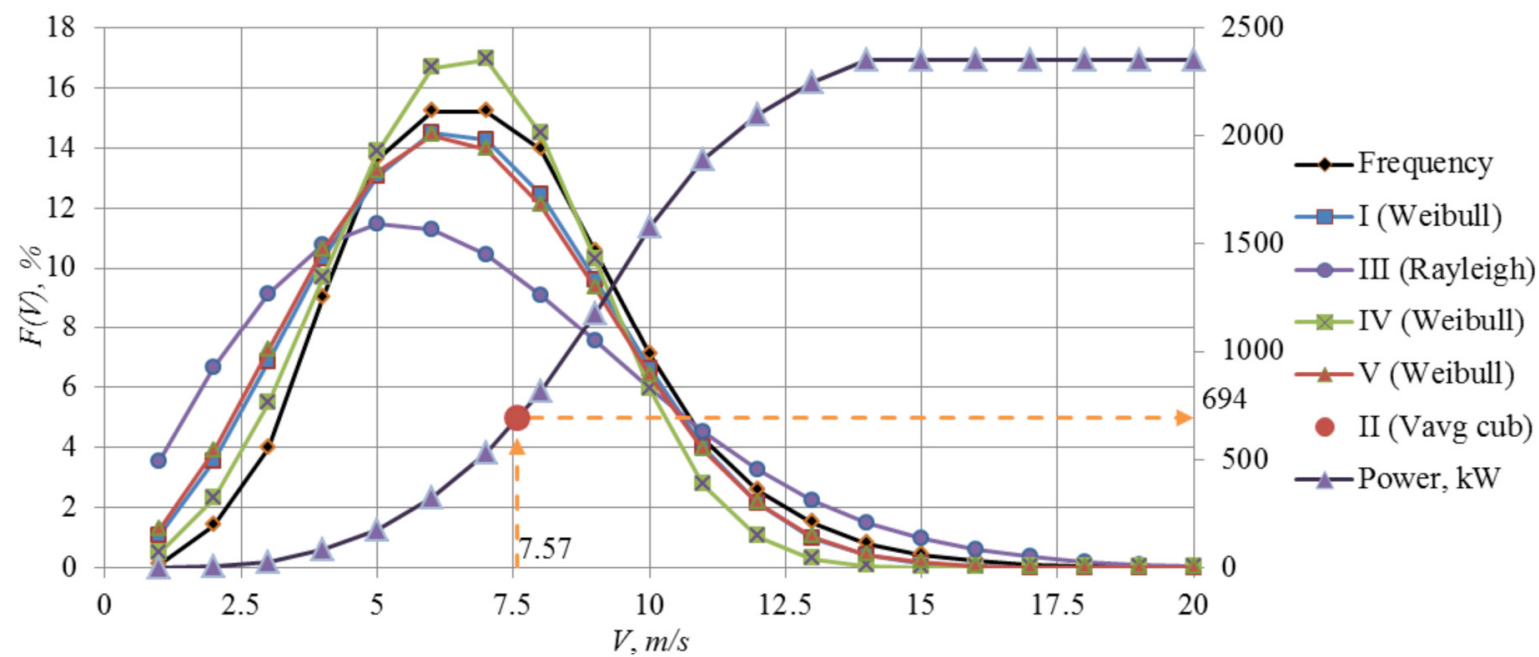

Fig. 4. Curves of wind speed frequency distributions $F(V)(\%)$, by Weibull and Rayleigh, the power curve $P(V)$ (kW) of WT type E82 for the height of $100(\mathrm{~m})$ and the corresponding value of average cubic wind speed $V_{\text {avg }}$ cub.

distributions of wind speed $V_{a v g}$ and $S D$ values give a good coincidence with the measurement results up to the height of $200(\mathrm{~m})$. However, for the cubic wind speed $V_{\text {avg cub }}$ the divergence of the approximating curve calculated using expression (4) from the real values increases with height.

\section{Comparison of methods for calculation AEP of WT}

The measuring complex Pentalum SpiDAR makes it possible to obtain a realistic picture for wind shear up to 200 (m); therefore, estimation of the model-related discrepancies in the calculated values of AEP by WTs located at different heights can be performed.

The algorithms of calculating the forecasted AEP for the heights of 80,100, 140, 180 and 200 (m) could systemized as follows:

- I (Weibull) - AEP determination by modelling the Weibull frequency distribution curves based on average wind speeds $V_{\text {avg }}$ and $S D$ for corresponding heights.

- II $\left(V_{\text {avg cub }}\right)$ - AEP determination by the value of average cubic wind speed $V_{\text {avg cub }}$ calculated using approximating function (4).

- III (Rayleigh) - AEP determination using approximating function (2) and calculating the Rayleigh wind speed frequency distribution.

- IV (Weibull) - AEP determination using approximating functions (2) and (3) in order to find $k$ - and $c$-factor values.

- V (Weibull) - AEP determination by approximating $k$ and $c$-factor values of Weibull frequency distribution function based on calculated $k$ and $c$ values at heights 30 , 40 and $50(\mathrm{~m})$.

The modelling of annual energy production using models $\mathrm{I} \div \mathrm{V}$ is performed taking the power curve $P(V)$ $(\mathrm{kW})$ of a $2300 \mathrm{~kW}$ wind turbine of the E82 type. Figure 4 shows the WT power curve, Weibull and Rayleigh curves of wind speed frequency distributions for 100 (m) height.
The value of average cubic wind speed for this height $V_{a v g}$ $c u b=7.57(\mathrm{~m} / \mathrm{s})$ and for this cubic wind speed corresponding power of generator is $694(\mathrm{~kW})$.

Analysis of curves in Figure 4 shows that the methods for AEP calculation considered above are not mutually connected by measurement parameters. This makes it possible to compare the modelling results with each other as well as with the calculation results obtained from measurements.

The total electricity production $W(\mathrm{GWh})$ to which the wind stream energy can be converted in a definite period of time is determined by the equality:

$$
W=\sum_{i=1}^{n}\left(P\left(V_{i}\right) \times F\left(V_{i}\right)\right),
$$

where the values of function $P\left(V_{i}\right)$ are corresponding to the power curve for the generator, while those of function $F\left(V_{i}\right)$ - to the Weibull's wind speed frequency distribution curve at the height of rotor hub and respective wind speeds $V_{i}(\mathrm{~m} / \mathrm{s})$.

Table 1 shows the results for forecast AEP (GWh) calculated using the data obtained during wind speed measurements as well as the results for forecast AEP according to variants $\mathrm{I} \div \mathrm{V}$ at heights of $80,100,140,180$ and $200(\mathrm{~m})$ above ground for the WT type E82. To determine the relative uncertainty of the results obtained at AEP calculation for different models the below shown expression is used:

$$
W_{\Delta}=\left(\frac{W_{\text {measur }}-W_{\text {mod }}}{W_{\text {measur }}}\right) \times 100(\%),
$$

where $W_{\text {measur }}$ is the forecasted AEP value (GW) calculated from the wind speed measurements, $W_{\text {mod }}$ is the forecasted AEP value $(\mathrm{GW})$ calculated from the results of modelling (models $\mathrm{I} \div \mathrm{V}$ ).

The results given in Table 1 are presented graphically in Figure 5, where it is seen that the relative deviation of AEP calculation $W_{\Delta}$ is decreasing with the height $h$ increasing in the Rayleigh model while in the Weibull model this value 


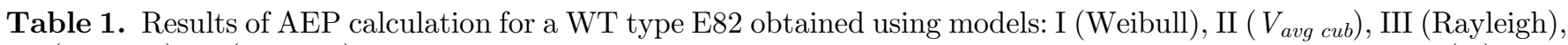
IV (Weibull), V (Weibull) and based on the wind speed measurements at heights 80, 100, 140, 180 and 200 (m).

\begin{tabular}{|c|c|c|c|c|c|c|c|c|c|c|c|}
\hline \multirow{3}{*}{$\begin{array}{l}h \\
(\mathrm{~m})\end{array}$} & \multicolumn{6}{|c|}{ AEP of WT (GWh) } & \multirow{2}{*}{\multicolumn{5}{|c|}{$\begin{array}{l}\text { Relative deviation of estimated AEP, } \\
\qquad W_{\Delta}(\%)\end{array}$}} \\
\hline & \multirow{2}{*}{$\begin{array}{c}W_{\text {measur }} \\
\mathrm{I}\end{array}$} & \multicolumn{5}{|c|}{$W_{m o d}$} & & & & & \\
\hline & & II & III & IV & $\mathrm{V}$ & I & II & III & IV & $\mathrm{V}$ & \\
\hline 80 & 4.72 & 3.98 & 4.7 & 4.73 & 3.94 & 4.18 & -15.6 & -0.3 & 0.4 & -16.6 & -11.4 \\
\hline 100 & 6.28 & 5.53 & 6.08 & 5.9 & 5.17 & 5.43 & -11.9 & -3.1 & -6.0 & -17.6 & -13.5 \\
\hline 140 & 8.88 & 8.05 & 8.73 & 7.81 & 7.59 & 7.65 & -9.4 & -1.6 & -12.1 & -14.6 & -13.9 \\
\hline 180 & 10.86 & 10.02 & 11.26 & 9.27 & 9.68 & 9.44 & -7.8 & 3.6 & -14.7 & -10.9 & -13.1 \\
\hline 200 & 11.72 & 10.95 & 12.45 & 9.87 & 10.56 & 10.2 & -6.6 & 6.2 & -15.7 & -9.9 & -13.0 \\
\hline
\end{tabular}

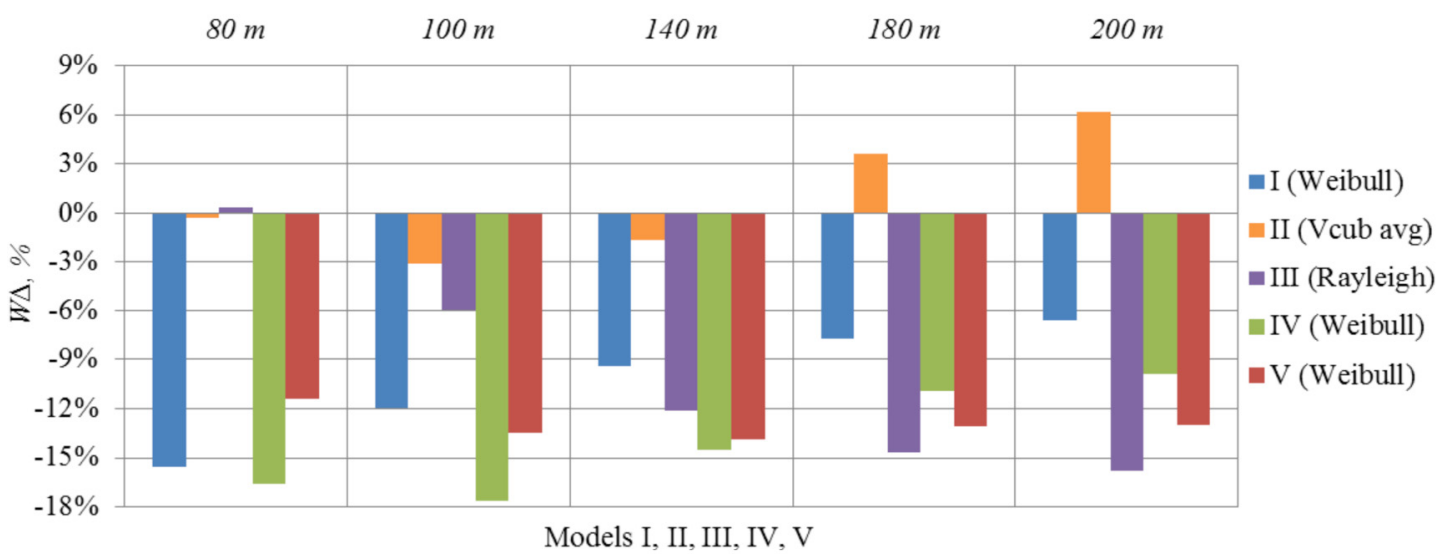

Fig. 5. Relative deflection $W_{\Delta}(\%)$ of AEP calculation based on models $\mathrm{I} \div \mathrm{V}$ for the heights $80,100,140,180$ and 200 (m).

depends but slightly on the height changes. As seen the results of $W_{\Delta}$ calculation for first and second models vary with height from +0.4 to $-17.6(\%)$.

At the same time, the relative error of the AEP calculation based on the average cubic wind speed is varying in the limits from -3.1 to $+6.2(\%)$. This peculiarity of the models' behaviour can be employed for increasing the precision of AEP calculation due to summing up and averaging predicted values.

\section{Conclusions}

Comparison of AEP calculation methods based on wind speed measurements on 30, 40 and $60(\mathrm{~m})$ height and wind shear extrapolation up to 200 (m) height shows that application of Rayleigh and Weibull models gives a result with relative deflection up to $-17(\%)$. The method of application of the value of average cubic wind speed gives relative deflection up to $6.2(\%)$.
This paper was supported by European Social Fund project "Assessment of wind energy potential in Latvia and environmental impact from wind energy installations", No. 2014/0010/1DP/ 1.1.1.2.0/13/APIA/VIAA/033.

\section{References}

1. J. Manwell, J. McGowan, A. Rogers, Wind energy explained: theory, design and application (John Wiley \& Sons Ltd., 2009), 689

2. Enercon wind energy converters: product overview 2010, Technical information. Version: July 2010: www.enercon.de/ p/downloads/EN_Productoverview_0710.pdf

3. V. Bezrukovs, V. Bezrukovs, A. Zacepins, Comparative efficiency of wind turbines with different heights of rotor hubs: performance evaluation for Latvia, J. Phys.: Conf. Ser. 524012113 (2014), 9 p. DOI:10.1088/1742-6596/524/1/ 012113

Cite this article as: Valerijs Bezrukovs, Aleksejs Zacepins, Vladislavs Bezrukovs, Vitalijs Komasilovs, Comparison of different methods for evaluation of wind turbine power production based on wind measurements, Renew. Energy Environ. Sustain. 1, 22 $(2016)$ 